\title{
Performance Analysis of Interferometric Noise Due to Unequally Powered Interferers in Optical Networks
}

\author{
J. C. Attard, J. E. Mitchell, Member, IEEE, and C. J. Rasmussen
}

\begin{abstract}
Interferometric crosstalk has been identified as the cause of performance limits in future transparent all-optical networks. A large number of studies have been conducted on this phenomenon using a vast array of evaluation techniques. However, most major studies have considered that although the interfering terms may differ in number, the power contribution that they all make will be identical for all interfering terms. Although this situation is easy to analyze, it does not necessarily represent the situation that is likely to occur in a real network, which will be constructed of nodes with different degrees of connectivity, quite possibly from different vendors, and therefore with differing crosstalk characteristics. This paper describes a study on the impact of unequally powered interfering terms using a rigorous analysis technique. To validate the use of the chosen technique, the paper begins by benchmarking a number of common evaluation techniques against empirically derived, experimentally verified noise performance formulas.
\end{abstract}

Index Terms-Interferometric noise, optical communications, optical crosstalk.

\section{INTRODUCTION}

I $\mathrm{N}$ recent years a number of authors have considered the impact of interferometric noise in multiwavelength optical networks [1]-[8]. Interferometric beat noise (also referred to as homodyne beat noise, phase-induced intensity noise, optical beat interference, or self-homodyne beat noise) arises from the beating on a square-law, envelope, photodiode receiver of the desired data signal and unwanted crosstalk signals derived from other data channels operating at nominally the same wavelength. These unwanted crosstalk signals may include multipath reflections of the main data signal or may be due to nonideal component performance. Square-law detection implies that the power attributed to the interferometric beat noise is much greater than that attributed to the incident crosstalk optical power. Indeed interferometric beat noise, which is signal dependent, has been reported to cause significant performance degradation, characterized by high power penalties and bit-error-rate (BER) floors [5].

Understandably, a large number of techniques have been applied to analyze this problem, covering a range from very simplistic first approximations [8] to highly rigorous and computationally intensive methods [3]. However, in order to simplify the analysis and/or the experimental setup, virtually all of these investigations applied to date have considered the presence of equally powered interferers at the receiver. Both [3] and [9] have

Manuscript received May 13, 2004; revised August 16, 2004

J. C. Attard and J. E. Mitchell are with the Department of Electronic and Electrical Engineering, University College London, London WC1E 7JE, U.K.

C. J. Rasmussen is with the Mintera Corporation, Lowell, MA 01852 USA.

Digital Object Identifier 10.1109/JLT.2005.844508 considered the effects of unequally powered interferers but only in very limited cases-where one interferer is of constant power and another (or set of others) is of varying power. A more realistic model of the actual interferer power distribution at the receiver in a deployed optical network is likely to be described by the presence of a set of interferers all of differing power at the receiver. In this paper, a detailed analysis of the behavior of an optical communications system in the presence of unequally powered interferers is provided.

The main optical, network, and component imperfections leading to the generation of interferometric beat noise are outlined hereafter.

1) Imperfect filtering or wavelength routing: Within all optical network components such as add-drop multiplexers or optical cross connects, some leakage is always present, albeit often small in magnitude. In an add-drop process for example, it is possible that a leakage component from the dropped channel is combined with the added channel, giving rise to interferometric beat noise at the receiver. Arrayed-waveguide gratings (AWGs) are also increasingly being deployed in optical networks due to their versatility to act as multiplexers, routers, and demultiplexers. In practical AWGs, imperfections in the device mean that some of the light power is also present on the other channels [7]. It is therefore possible that when the AWG is used to route channels of a similar wavelength from different input channels to different output channels, crosstalk that leads to inband interferometric beat noise occurs.

2) Reflections: Refractive-index discontinuities in the optical fiber due to splices, connectors, couplers, and other passive devices are known to cause reflections of the optical signal. In [8], it has been shown that an air gap between two connector end faces can reflect as much as $22.4 \%$ of the incident optical power. A cascade of two or more discontinuities in the system will permit multiple reflections to be caused. These will beat on the photodiode receiver and generate interferometric noise.

3) Fiber nonlinear effects: Fiber nonlinear effects, namely in the form of stimulated Brillouin scattering (SBS), Rayleigh scattering, or four-wave mixing (FWM) [10], may also give rise to interferometric beat noise.

Previously, it has been indicated in the literature that the Gaussian approximation (GA) tends to provide erroneous highly pessimistic results when the number of interferers is 
TABLE I

INTERFEROMETRIC NOISE EVALUATION TECHNIQUES

\begin{tabular}{|c|c|c|}
\hline Technique & Description & Ref \\
\hline $\begin{array}{l}\text { The Gaussian } \\
\text { approximation }\end{array}$ & $\begin{array}{l}\text { Leads to a closed form power penalty estimation formula. } \\
\text { Typically used as a first approximation. Accurate for a } \\
\text { large number of interferers. }\end{array}$ & {$[5],[8]$} \\
\hline Inner eye closure & $\begin{array}{l}\text { Obtains a pessimistic penalty estimate (upper bound) from } \\
\text { the inner eye closure. Simple to apply, poor results if the } \\
\text { range of the interfering variable }>>\text { standard deviation e.g. } \\
\text { multiple interferers. }\end{array}$ & $\begin{array}{l}{[20],} \\
{[21]}\end{array}$ \\
\hline $\begin{array}{l}\text { Numerical } \\
\text { Convolution }\end{array}$ & $\begin{array}{l}\text { A numerical method based on the convolution of the } \\
\text { interferometric noise PDFs. Accurate results, but hard to } \\
\text { apply numerically due to the need to integrate infinite tails. }\end{array}$ & $\begin{array}{l}{[22],} \\
{[23]}\end{array}$ \\
\hline $\begin{array}{l}\text { The Gauss } \\
\text { quadrature rule }\end{array}$ & $\begin{array}{l}\text { BER may be estimated to any required degree of accuracy } \\
\text { by using an appropriate number of moments of the } \\
\text { interferometric noise PDF. Applied in }[19] \text { and compared } \\
\text { with other estimation techniques. Similar level of accuracy } \\
\text { to the saddlepoint approximation. }\end{array}$ & [24] \\
\hline $\begin{array}{l}\text { The Chernoff } \\
\text { Bound }\end{array}$ & $\begin{array}{l}\text { Simple bounding technique based on the moment } \\
\text { generating function. Very easy to apply, not always a very } \\
\text { tight bound. }\end{array}$ & [25] \\
\hline $\begin{array}{l}\text { The Modified } \\
\text { Chernoff Bound }\end{array}$ & $\begin{array}{l}\text { MCB uses the knowledge of the presence of a Gaussian } \\
\text { component in the noise to improve the Chernoff bound. } \\
\text { It is shown that the MCB provides very accurate results. }\end{array}$ & $\begin{array}{l}{[25],} \\
{[26]}\end{array}$ \\
\hline $\begin{array}{l}\text { The Saddlepoint } \\
\text { approximation }\end{array}$ & $\begin{array}{l}\text { Uses the saddlepoint value of the integrand of the MGF to } \\
\text { approximate the error probability. Produces a good } \\
\text { approximation in most cases. }\end{array}$ & [27] \\
\hline $\begin{array}{l}\text { Gram-Charlier } \\
\text { series } \\
\text { approximation } \\
\text { and other series } \\
\text { expansions }\end{array}$ & $\begin{array}{l}\text { A correction of the Gaussian approximation, expresses an } \\
\text { arbitrary PDF as an infinite series whose leading term is a } \\
\text { Gaussian distribution. All the series coefficients can be } \\
\text { evaluated from the moments of the random variable [23]. } \\
\text { Applied in [3] where it was shown that it provides good } \\
\text { results even for a small number of interferers. }\end{array}$ & $\begin{array}{l}{[3],} \\
{[28]}\end{array}$ \\
\hline $\begin{array}{l}\text { Hermetain } \\
\text { Polynomials }\end{array}$ & $\begin{array}{l}\text { Used to analyse the single interferer case and obtain a } \\
\text { closed form BER formula, truncation may limit accuracy. }\end{array}$ & [24] \\
\hline Simulation & $\begin{array}{l}\text { The detailed simulation of a system modelled by the } \\
\text { accurate characterisation of all network components may } \\
\text { be used to obtain a BER estimate. Time consuming for } \\
\text { large networks }\end{array}$ & [29] \\
\hline $\begin{array}{l}\text { Adaptive } \\
\text { Importance } \\
\text { Sampling } \\
\end{array}$ & $\begin{array}{l}\text { Use of Monte Carlo simulation techniques with weighted } \\
\text { variances }\end{array}$ & {$[30]$} \\
\hline
\end{tabular}

low [11]. In this paper, it will be demonstrated that this can also be true when the interfering power is distributed unequally among a higher number the interferers. This is explained by examining in detail the counterintuitive trends produced by the for a varying numbers of interferers. To provide more accurate results, the work in this paper is based on an analysis using a rigorous statistical method. A number of techniques are available (see Table I), many of which are based on moment-generating functions (MFGs) and produce results of comparable accuracy (e.g., the saddlepoint approximation and modified Chernoff bound (MCB) [12]). However, the MCB [13] was selected due to its computational efficiency. It is shown that this technique is very appropriate for the analysis of interferometric noise arising from unequally powered interferers. Finally, the performance estimates obtained using this rigorous approach have been benchmarked against experimentally verified simulation results showing that the accuracy of the results obtained using the MCB are practically indistinguishable from the experimentally verified benchmark results.

\section{Modeling Interferometric NoISe Due to MultiPle INTERFERERS}

Consider the mixing on a photodiode receiver of an optical data signal whose electric field is represented by $E_{s}(t)$, with $N$ crosstalk interferers whose electric field is represented by
$\sum_{x=1}^{N} E_{x}(t)$. The total electric-field incident on the photodiode receiver $E_{\text {total }}(t)$ is modeled as

$$
\begin{aligned}
E_{\text {total }}(t)= & E_{s}(t)+\sum_{x=1}^{N} E_{x}(t) \\
E_{\text {total }}(t)= & {\left[\vec{\rho}_{s} \sqrt{m_{s}(t) P_{s}} e^{j\left(\omega_{s} t+\phi_{s}(t)\right)}\right.} \\
& \left.+\sum_{x=1}^{N} \vec{\rho}_{x} \sqrt{\varepsilon_{x} m_{x}(t) P_{s}} e^{j\left(\omega_{x} t+\phi_{x}(t)\right)}\right]
\end{aligned}
$$

where $\vec{\rho}$ expresses the state of the polarization vector, $P_{s}$ is the power of the desired optical signal at the photodiode., $\omega$ the optical angular frequency, $\phi(t)$ the optical phase, $m(t)$ the binary symbols forming the ON-OFF keying (OOK) message where $m(t) \in\{r, 1\}(0<r<1)$, with $r$ accounting for the extinction ratio and $\varepsilon_{x}$ is the relative power in the $x^{\text {th }}$ crosstalk term $\left(\varepsilon_{x}=P_{x} / P_{s}.\right)$.

The subscripts $s$ and $x$ denote signal and crosstalk, respectively. Following square-law detection on a photodiode, and assuming a normalized responsivity, the photocurrent $i(t)$ is proportional to

$$
\begin{aligned}
& i(t) \propto\left|E_{\text {total }}(t)\right|^{2} \\
& i(t) \propto P_{s}(t)\left[m_{s}(t)+\sum_{x=1}^{N} \varepsilon_{x} m_{x}(t)\right. \\
& +2 \sum_{x=1}^{N}\left(\vec{\rho}_{s} \times \vec{\rho}_{x} \sqrt{\varepsilon_{x} m_{s}(t) m_{x}(t)}\right. \\
& \left.\quad \times \cos \left\{\left(\omega_{s}-\omega_{x}\right) t+\phi_{s}(t)-\phi_{x}(t)\right\}\right) \\
& +2 \sum_{x=1}^{N-1} \sum_{j=x+1}\left(\vec{\rho}_{x} \times \vec{\rho}_{j} \sqrt{\varepsilon_{x} \varepsilon_{j} m_{x}(t) m_{j}(t)}\right. \\
& \times \cos \left\{\left(\omega_{x}-\omega_{j}\right) t+\phi_{x}(t)\right. \\
& \left.\left.\left.-\phi_{j}(t)\right\}\right)\right] .
\end{aligned}
$$

It can be seen that the photocurrent is composed of the following four components:

1) the data signal;

2) an additive crosstalk signal (also referred to as the classical crosstalk);

3) a beat term that exhibits a co-sinusoidal dependence on the relative wavelength and phase difference;

4) in the multiple interferer case, a fourth component is present representing the crosstalk to crosstalk beating.

Equation (4) highlights several important properties of interferometric noise:

a) The beat noise contribution is far larger than that due to the additive crosstalk. For example, if the data contribution is normalized and that of the crosstalk is $10 \%$, then the beat noise contribution spans $\pm 63 \%$ [34]. Beat noise is therefore usually the dominant noise when it is present to any significant level.

b) The beat noise magnitude is dependent on the relative polarization alignment of the data and crosstalk signals. When the two signals are orthogonally polarized relative 
to each other there is in theory no beat noise. In [14], the polarization statistics of interferometric noise in optical networks have been investigated. It was found that systems with randomly polarized fields display a statistical preference for near worst-case operation.

c) Increasing the signal power does not change the relative level between the signal and the beat noise magnitudes; consequently, the optical beat noise may dominate over shot or thermal noise in the receiver and lead to an error floor.

d) If the data and the crosstalk signal are at the same wavelength, then the additive crosstalk and the beat noise both fall inband. If the data and the crosstalk are on different wavelengths and the wavelength difference is much larger than the receiver electrical bandwidth, then the beat noise falls out of band and only the additive classical crosstalk is inband.

e) Inband crosstalk: Following the classification presented in [34], when the data and the crosstalk signals originate from the same laser source which exhibits a coherence time of $\tau_{c}$, and the crosstalk is a replica of data signal delayed by $\tau$, then the following is applicable.

i) If $\tau<\tau_{c}$, coherent beat noise crosstalk occurs. In the limit, $\tau=0$, which is characterized by the absence of phase noise. It must be appreciated that if $\tau$ is constant the beat noise term is static and may be determined.

ii) If $\tau \gg \tau_{c}$, incoherent beat noise crosstalk occurs. In the case of a continuous-wave $(\mathrm{CW})$ distributed feedback (DFB) laser operating at $1 \mathrm{~mW}$, the coherence time $\tau_{c}$ is of the order of nanoseconds; thus, the incoherent region is achieved with a path length difference $>10 \mathrm{~m}$. Incoherent beat noise may also arise from the beating of signals originating from different lasers operating on the same wavelength.

iii) If $\tau \sim \tau_{c}$, partially incoherent beat noise crosstalk occurs. This crosstalk falls between the two extremes of coherent and incoherent beat noise crosstalk. The resulting noise is very difficult to analyze since its properties depend on the exact value of the delay $\tau$.

Inband crosstalk cannot be removed as the signal and the crosstalk are on the same wavelength. In complex optical networks, its deteriorating effect is further enhanced due to its accumulative behavior.

f) Out-of-band crosstalk: When the beat frequency $\left(\omega_{s}-\right.$ $\left.\omega_{x}\right) / 2 \pi$ between the data and the crosstalk signals is much greater than the receiver bandwidth, then the beat noise falls out of band where, following detection, it may be removed by electronic filtering and only additive components remain. If the beat frequency between the two signals is of the order of the receiver bandwidth, then the beat noise falls totally or partially inband giving rise to incoherent beat noise crosstalk. An estimate of the signal-to-interference ratio arising from optical beat interference between Lorentzian-shaped optical sources has been derived in [15], [16]. Out-ofband crosstalk may be minimized by utilizing tighter or cascaded filtering.

We will now review methods that are available to evaluate the impact of interferometric noise in optical networks.

\section{INBAND INTERFEROMETRIC NOISE ESTIMATION METHODS}

Interferometric noise estimation methods applied in research to date range from assuming a Gaussian probability density function (pdf) for the interferometric noise [17], thereby enabling a quick penalty estimation, to deriving an accurate model of the actual interferometric noise phenomena at the cost of a lengthier process involving more accurate statistics [6].

Preliminary work in this area has assumed that it is acceptable to approximate the interferometric noise pdf to that of a Gaussian pdf, citing the central limit theorem as justification. It has often been applied since this approximation leads to a closed form expression for the power penalty [8]. However, the noise pdf that actually determines the system BER is obtained by the convolution of the pdf's of the individual interfering components, including the interferometric noise and the receiver Gaussian thermal noise. The inaccuracy of the method in this case is not surprising since the arc-sinusoidal pdf describing the interferometric noise is strictly bounded, and the assumption that it is Gaussian with infinite tails is an oversimplification especially for cases where the number of interferers is low. A summary of the principal interferometric noise analysis techniques used in the literature are outlined in Table I together with some key references.

\section{ANALYSIS OF THE IMPACT OF INTERFEROMETRIC NOISE ON NETWORK PERFORMANCE}

We begin by formulating a set of generic equations to describe the impact of interferometric noise in an optical system. We will demonstrate the formulation of both the GA and the MCB. Although versions of both of these evaluation methods that do not take into account symbol states can be used, here we will only consider those that are symbol conditioned, since this is more accurate representation. Symbol conditioning evaluates the penalty for all possible combinations of symbol states of the interferers for each symbol state of the desired signal rather than assuming that all interferers are constantly " 1 ", e.g., CW interferers.

An OOK data signal mixing on a photodiode together with homodyne crosstalk caused by $N$ interferers is considered. The following assumptions are made so that an expression for the normalized decision variable photocurrent, shown in (5), can be derived.

1) The bit alignment between the signal and crosstalk interferers is perfect.

2) The optical phase difference remains constant within 1-b duration.

3) Post detection filter is an integrating filter, integrating between 0 and $T$, where $T$ is the bit period. Due to the normalization process, results are independent of bit rate.

4) The impact of additive (or classical) crosstalk is neglected since this has an insignificant impact on the 
performance of the system in the presence of interferometric beat noise.

5) Both the signal and all interfering terms have identically aligned polarization states for worst-case impact.

6) All beat components will fall within the bandwidth of the receiver-i.e., signal and the interferers will all be operating on the same wavelength. Furthermore, for a high-bit-rate system, it is reasonable to assume that the receiver bandwidth will be much greater than the linewidth of the optical source; therefore, all the interferometric noise will fall inband. This assumption may not be valid for interference between isolated " 1 " bits generated by a directly modulated laser system. In such a case the interferometric noise may fall outside the receiver bandwidth. Interference for sequences with several consecutive " 1 " bits would, however, cause all the power to fall inband. This assumption is therefore a worst-case assumption. On the other hand, if the chirp of the laser is small and the data rate is high, the chirp frequency may fall inside the receiver bandwidth. This will result in a performance that is very similar to external modulation, which has negligible chirp.

7) Anidealp-i-n receiver: For p-i-n receivers, the shot noise due to the signal itself can generally be considered to be negligible. In the absence of other signal-dependent noise sources the noise variances and distributions of the signal photocurrent are assumed to be the same for both ones and zeros. The optimum decision threshold is therefore midway between the expected signal levels. Interferometric noise is a signal dependent noise, thus the optimal decision threshold is not at the midpoint of the eye. In this paper, we present results based on receivers that have both a midway decision threshold and an optimized decision threshold. The computed results cannot therefore be directly ported to Avalanche Photodiode (APD) systems since in such systems the optimum decision threshold must also take into consideration the APD excess multiplication noise [8]. A similar situation is true of optically preamplified receivers, where signal-spontaneous beat noise will be the dominant noise contribution of the amplifier, which will result in an additional signal-dependent contribution. This will degrade the performance of the system. A method of extending the model to include such terms has been demonstrated in [18].

8) An ideal extinction ratio is assumed as justified in [19].

9) The effect of crosstalk-crosstalk beating has been assumed to be negligible. It can be shown that when symbol condition is included in the model, the effect of crosstalk-crosstalk beating is minimal [35] but is exaggerated when $\mathrm{CW}$ interferering sources are considered, as was demonstrated by Kamalakis et al. [37].

10) The instantaneous phase difference $\phi_{d}(t)=\phi_{s}(t)-$ $\phi_{k}(t)$ between the phase of the optical data signal and the phase of the optical $k$ th interfering signal at time $t$ is represented by a uniform random variable distributed in $[0 ; 2 \pi]$.
Following these assumptions, the normalized decision variable photocurrent is equal to

$$
i(t)=P_{s}\left[m_{s}(t)+2 \sum_{k=1}^{N} \sqrt{\varepsilon_{k} m_{s}(t) m_{k}(t)} \cos \left[\phi_{d}(t)\right]+\right]
$$

where the subscript $s$ denotes the desired signal, $k^{\text {th }}$ interfering signal, and other terms as defined previously.

To include symbol conditioning, the model is refined by applying binomial symbol conditioning on the interfering signals for a more accurate representation. This leads to the following expressions for symbol conditioned GA (6) and for the symbol conditioned MCB (7). It is assumed that " 0 " and " 1 " bits appear with equal likelihood and that all interfering terms are of equal power.

The symbol-conditioned Gaussian approximation (SCGA)[38] is

$$
\begin{array}{r}
\mathrm{BER} \cong\left(\frac{1}{2}\right)^{N+1} \sum_{k=0}^{N} C_{k}^{N}\left\{Q\left[\frac{P_{s}-D}{\sqrt{\sigma_{\mathrm{th}}^{2}+\sigma_{\mathrm{IN}_{k}}^{2}}}\right]\right\} \\
+\left(\frac{1}{2}\right) Q\left[\frac{D}{\sqrt{\sigma_{\mathrm{th}}^{2}}}\right] .
\end{array}
$$

The symbol-conditioned MCB [25] is

$$
\begin{aligned}
& \mathrm{BER} \leq \operatorname{MCB}(s)=\frac{e^{\frac{\left(s \sigma_{\mathrm{th}}\right)^{2}}{2}}}{2 s \sigma_{\mathrm{th}} \sqrt{2 \pi}} \\
& \times\left[\left(\frac{1}{2}\right)^{N} \sum_{k=0}^{N} C_{k}^{N} M_{\mathrm{IN}}(s)^{k} e^{-s\left(P_{s}-D\right)}+e^{-s D}\right], \quad s>0
\end{aligned}
$$

where $Q$ is an approximation to the area under the Gaussian tail $Q(x) \approx\left(1 / \sqrt{2 \pi x^{2}}\right) e^{-x^{2} / 2}, \sigma_{\text {th }}^{2}$ is the variance of the receiver thermal noise (taken to give a base BER of $10^{-9}$ in the absence of other noise contributions), $D$ the decision threshold, and $\sigma_{\mathrm{IN}}^{2}=2 \varepsilon P_{s} N$ is the interferometric noise variance which is only present when both signal and interferer at carrying a data " 1 ". $M_{\mathrm{IN}}(s)=I_{0}\left(2 s \sqrt{\varepsilon} P_{s}\right)$ is the moment generating function of the interferometric noise where $I_{0}$ is the modified Bessel function first kind, zero order, and $C_{k}^{N}=N ! / k !(N-k)$ !.

Theoretical investigations using both the saddlepoint approximation [32] and the MCB [33] have noted that the penalty caused by a given total crosstalk power increases when this power is distributed among an increasing number of crosstalk signals. This is a phenomenon that is not predicted by a nonsymbol-conditioned since this is conditioned only by the total crosstalk power irrespective of the individual interferer contribution to that total.

As demonstrated in Fig. 1, the nonsymbol-conditioned GA is identical for all $N$ as it is only conditioned on the total interfering power which, in the absence of symbol conditioning, is constant. Introducing symbol conditioning to the to investigate the impact of dividing a given total crosstalk power among a varying number of interferers in [17] produced a surprise result where, contrary to intuition, it was predicted that the performance of the system improves as the given crosstalk power is divided among more interferers. When the more rigorous MCB-based approach was used, the contrary trend was predicted. 


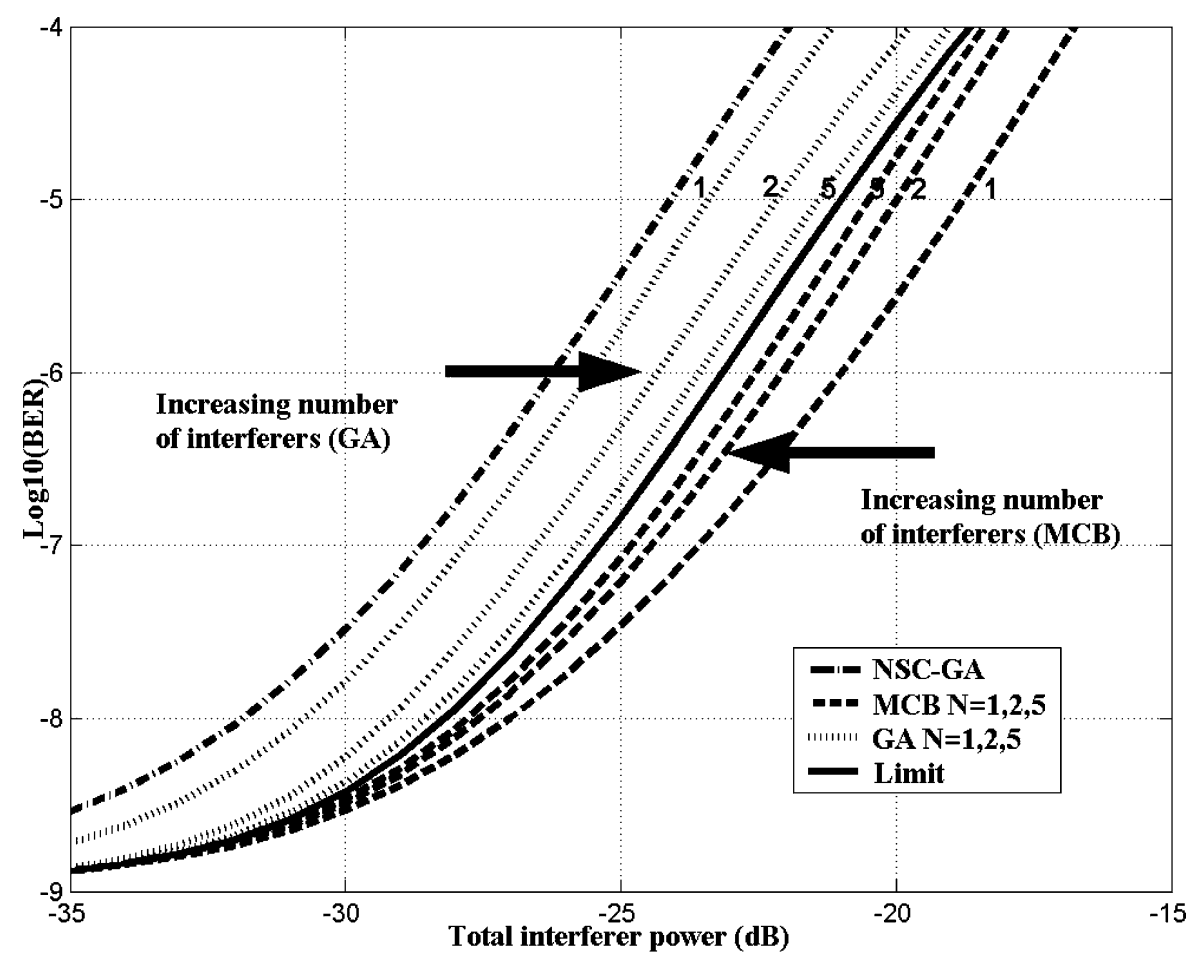

Fig. 1. Comparison of the nonsymbol-conditioned (dotted-dashed line) and symbol-conditioned GA (dotted line) and MCB (dashed line). The limit converged upon by the symbol-conditioned GA and MCB. All three nonsymbol-conditioned lines overlap with the symbol-conditioned GA for a single interferer.

Here we explain this contradictory behavior of the GA and the MCB when they are used to analyze the performance of an optical system with equally powered interferers. The results obtained when investigating how the techniques behave when analyzing a system where a given total interfering power is divided among one, two, and five equally powered interferers are presented. In the single interferer case $(N=1)$, all the interfering power is contributed by a single interferer of normalized power. When the number of interferers is equal to two $(N=2)$, each will contribute a power of 0.5 . When the number of interferers is equal to five $(N=5)$, each interferer will contribute a power of 0.2. In Fig. 1 the results for binomial symbol conditioning of the interferers are illustrated using the GA and the MCB. A midway decision threshold has been assumed.

It is observed that the GA and the MCB predict opposing trends as the number of interferers is increased. The GA predicts that the performance improves when a given interfering power is divided among an increasing number of interferers. This trend was first noted by Legg [17] who concluded that the system may tolerate more total crosstalk when the crosstalk is distributed over more terms.

On the other hand, the MCB predicts the opposite trend. In [6] it was concluded that the system may therefore tolerate less crosstalk when the crosstalk is distributed over more terms due to the increasing tail of the probability distribution and attributed the counter trend to be due to the inaccuracy of the GA when applied to a small number of interferers. The largest discrepancy between the results predicted by the GA and the MCB occurs for a single interferer. It may also be observed that the results estimated by both the GA and the MCB converge to a common prediction as the number of interferers increases.

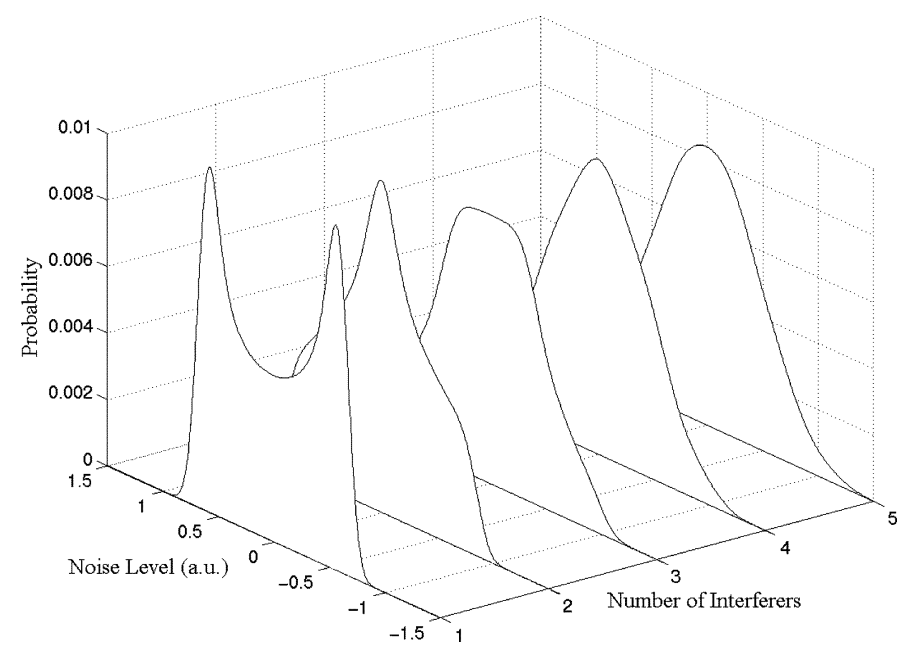

Fig. 2. Evolution of the noise pdf from one interferer to five interferers including a Gaussian noise contribution.

Using a moment-generating-function-based technique, which considers the full statistical representation of the noise, we investigate how the resulting noise pdf evolves as it is caused by an increasing number of interferers. With a single interferer, the pdf is still reasonably tightly bounded despite the inclusion of a Gaussian noise term to represent thermal noise. Inclusion of more interfering terms corresponds to the multiple convolution of the arc-sinusoidal pdf which increases the tails of the distribution. As it is these tails that determine the performance of the system, it is intuitive to assume that an increase in the tails will have a detrimental effect on the BER performance. This evolution is demonstrated in Fig. 2, which follows similar trends to those predicted in [36]. 


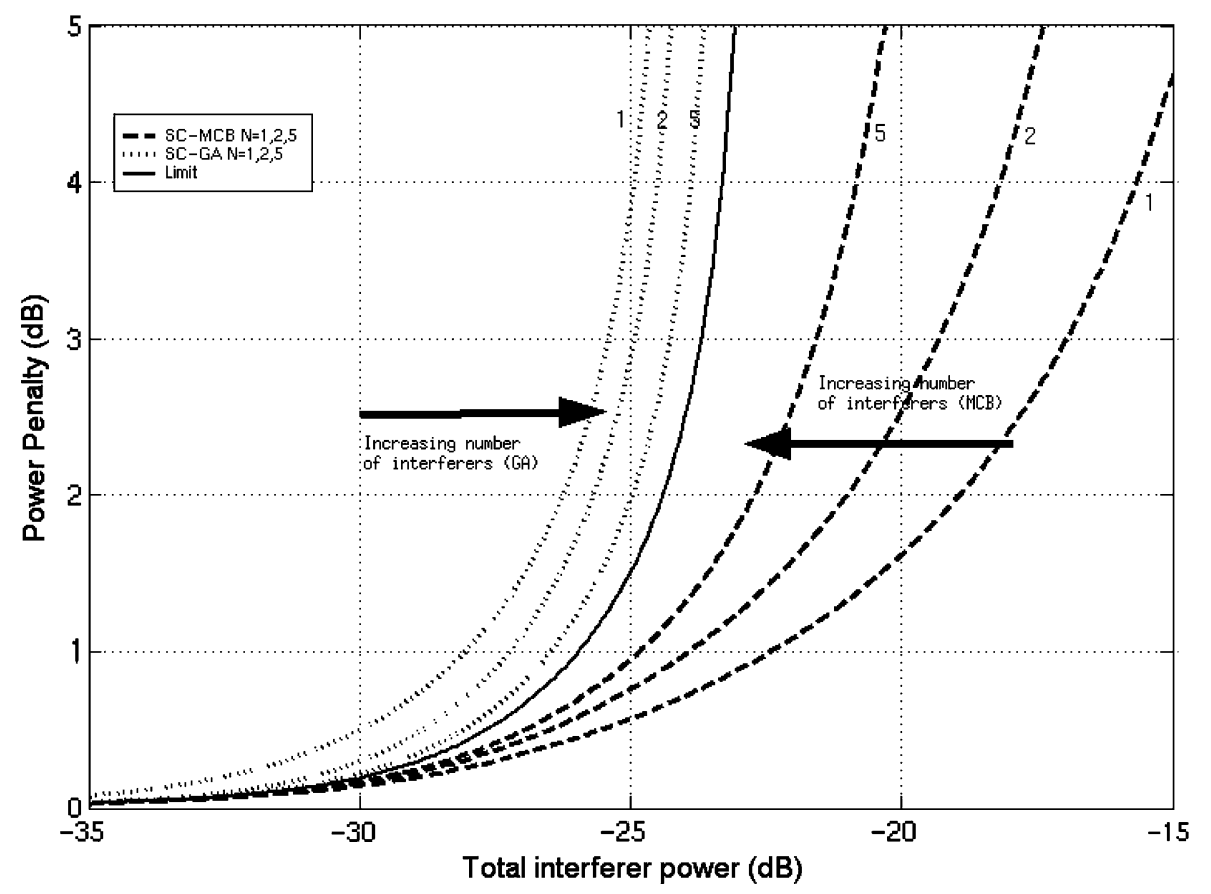

Fig. 3. Power penalty for equally powered interferers (midway decision threshold).

The power penalties estimated using the GA and MCB are illustrated in Fig. 3, where the opposing trends predicted by the GA and the MCB are again observed. The existence of BER floors is evident as illustrated by the asymptotic nature of the penalty curves. It is estimated that for the single interferer case, there is approximately a 9-dB difference between the GA and MCB techniques in the estimated crosstalk at which the BER floor occurs. This difference becomes progressively smaller as the number of interferers is increased, until both results converge to within $1 \mathrm{~dB}$ for approximately $N>6$. It is also noted that the penalty due to the interferometric noise is very close to zero when the total interfering power is small.

\section{BenCHMARKING THE MODIFIEd CHERNOFF BOUND}

Although it has been concluded that the performance of the MCB is better than the GA, this has often been on intuitive grounds due the more exacting statistical nature of the bound compared to the . It is important, however, that this bound is validated in this regime of operation to ensure that it is tight enough to produce satisfactory results. Recently empirical formulas have been developed that state the crosstalk penalty as a function of the total crosstalk power, the number of crosstalk contributions, and the signal extinction ratio [4]. The empirical formulas are derived from results that have been obtained via intensive numerical simulation based on the numerical convolution of the interferometric noise pdf's. These results were experimentally verified by Rasmussen $e t$ al. [4].

The empirical formulas have been published for an optimized decision threshold with the penalties produced deviating less than $0.2 \mathrm{~dB}$ from those obtained by experiment. The correct ${ }^{1}$ for-

${ }^{1}$ The formulas were incorrectly reproduced in the original publication [4]. mulas for a p-i-n receiver with an optimized decision threshold are

$$
\begin{aligned}
& \text { Penalty }[\mathrm{dB}]=-A \log _{10}\left(1-e^{b\left(X_{\mathrm{dB}}-X_{o}\right)}\right) \\
& \begin{array}{l}
A=4.6+\left(22+63 r_{\mathrm{dB}}^{-0.59}\right) N^{-1.45} \\
b=0.163+\left(0.0656-0.00218 r_{\mathrm{dB}}\right)\left(1-\frac{1}{N}\right) \\
X_{o}=\frac{\left(-23.36-228 r_{\mathrm{dB}}^{-1.295}\right)}{\left\{1+\left[N\left(0.81+10.5 r_{\mathrm{dB}}^{-1.54}\right)\right]^{\left(-1.68+2.05 r_{\mathrm{dB}}^{-0.834}\right)}\right\}} \\
\quad+9.32+221 r_{\mathrm{dB}}^{-1.55}
\end{array}
\end{aligned}
$$

where $r_{\mathrm{dB}}$ is the extinction ratio defined as $-10 \log (r)$ where $r$ is as previously defined and $X_{\mathrm{dB}}$ is the total relative crosstalk power in decibels and $N$ is the number of interferers. Fig. 4 illustrates the application of the empirical formulas for an extinction ratio of $30 \mathrm{~dB}$ for the cases of a one, two and five interferers with results obtained for both optimized and a mid-eye decision threshold. The error bars on the graphs for the optimized decision threshold show the extent of the $0.2-\mathrm{dB}$ variation from these results and those obtained by experiment. It is clearly seen that when a given total interfering power is divided among an increasing number of interferers, the power penalty increases as predicted by the MCB. The results for the mid-eye decision threshold have been obtained via numerical simulation. Only the optimal decision threshold results have been experimentally verified. 


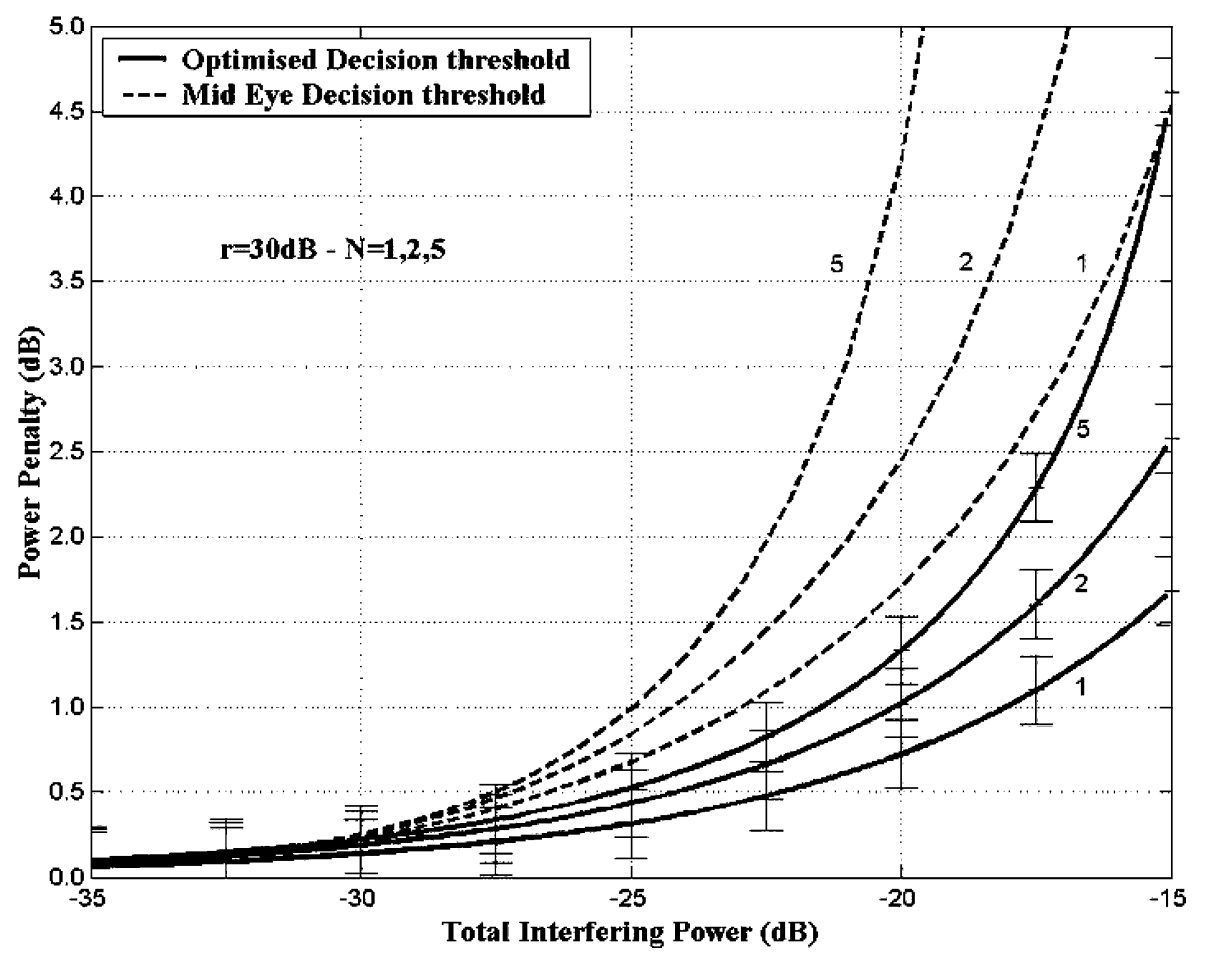

Fig. 4. Plot of the numerically simulated results with the optimized decision threshold and the mid-eye decision threshold for one, two and five interferers. The extinction ratio is $30 \mathrm{~dB}$.

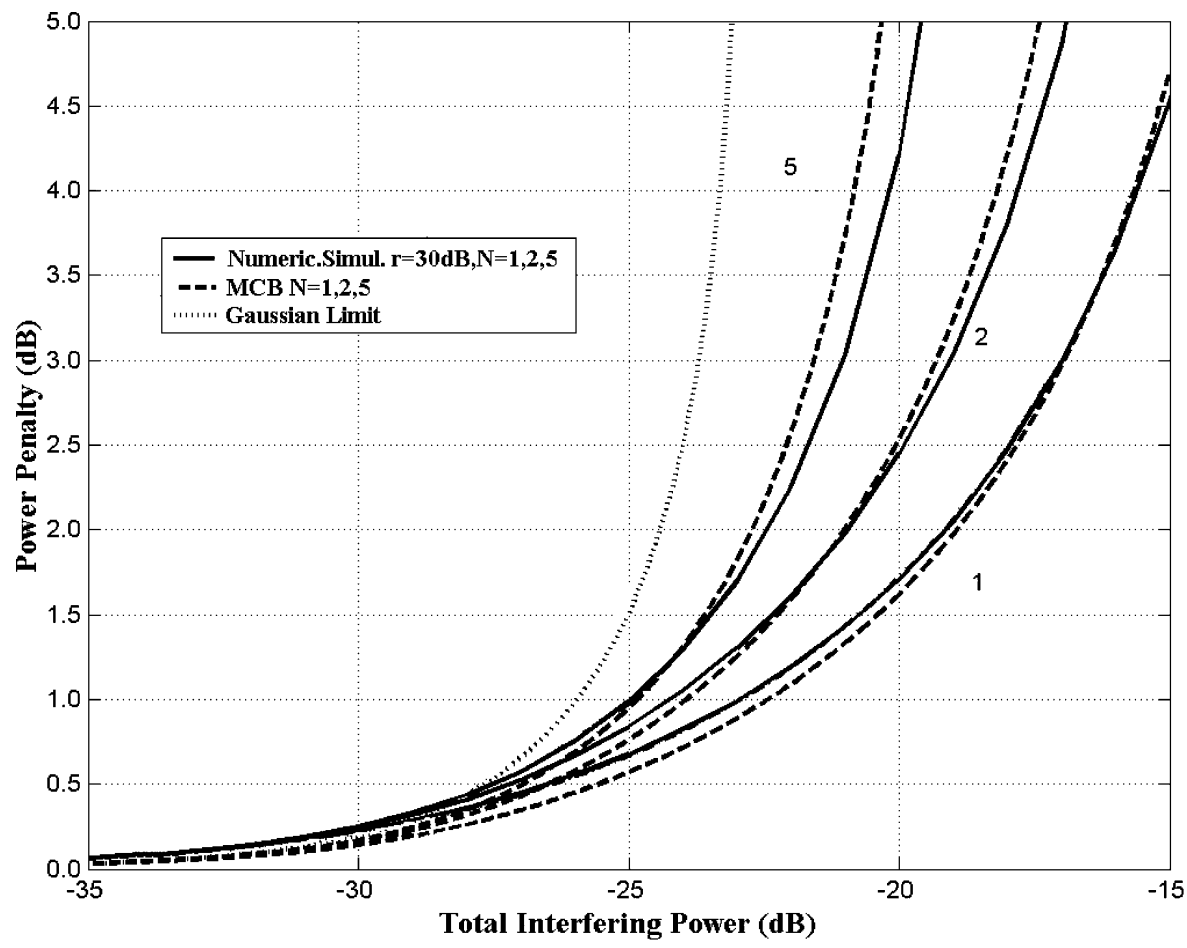

Fig. 5. Benchmarking numerically simulated (dashed) and MCB derived results (solid lines) for one, two, and five interferers. The Gaussian central limit is also shown.

In Fig. 5, the results obtained using the MCB are benchmarked with the numerically simulated results for a mid eye decision threshold. It is seen that the numerically simulated results are in close agreement with the MCB results in general, with the results virtually identical for a single interferer. In Fig. 6, the results obtained using the MCB with an optimized decision threshold are benchmarked with the experimentally verified empirical formulas. It is clearly seen that the results are in close agreement falling within the error bounds for both the single interferer and two interferer cases. In all benchmarked cases-be- 


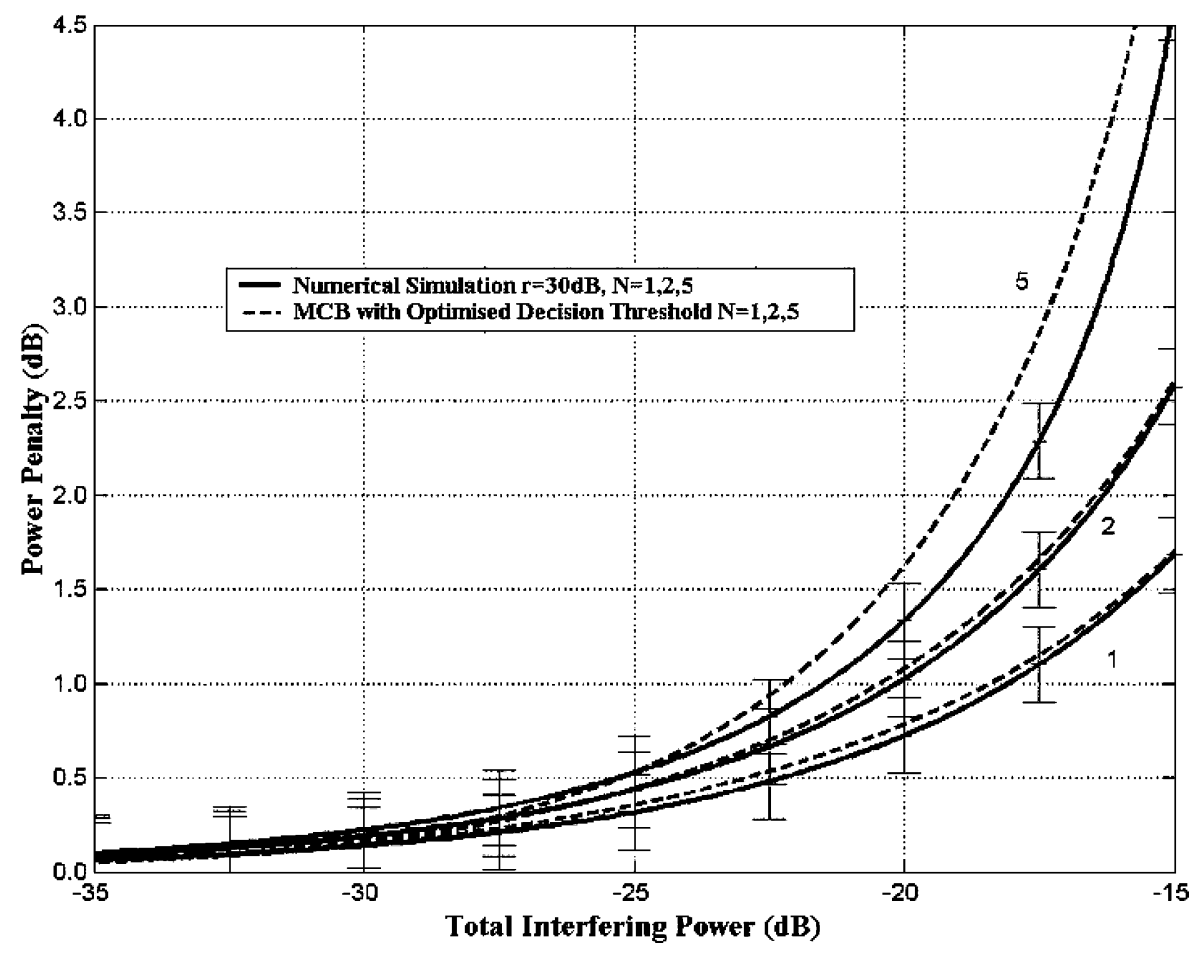

Fig. 6. Benchmarking simulated/experimental (solid) and optimized MCB derived results (dashed) for one, two, and five interferers.

sides highlighting the accuracy of the MCB-the trend predicted when the total interfering power is divided among more interferers has also been confirmed.

This result has direct implications on how the performance of optical access systems in the presence of interferometric noise is estimated since it is very likely that crosstalk conditions on these networks are such that the total interfering power is carried by a number of unequally powered interferers.

\section{INTERFERERS OF UNEQUAL POWER}

The motivation for a detailed investigation into this effect is due to the fact that the statistical distribution of the interferer power on a deployed wavelength-routed optical network is more likely to be a contribution of interferers of different strength rather than a uniform one. The unequally powered interferer case is therefore a more realistic representation of typical conditions.

In this section, we will evaluate the results obtained when a total interfering power is divided among a varying number of interferers in a nonuniform way. A midway decision threshold has been assumed.

Although the central limit theorem is frequently relied upon to justify the use of the GA in the previous section it has been demonstrated that when a total interfering power is carried by a small number of interferers a lower penalty is incurred. A logical extension of this conclusion leads to the hypothesis that if a large number of interferers of unequal power are considered then for the central limit theorem to be safely applied no small number, or a single interferer must be dominant. In this section, we will consider the impact that a varying numbers of unequal interferers has on the overall system performance. This is demonstrated by using the MCB to investigate the performance of an optical network in the presence of interferometric noise. The results obtained are also compared with those obtained using the GA.

To illustrate the significance of various power distributions among the interferers, a skewing factor is introduced to determine the power in each successive interfering term. This factor is defined as

$$
\alpha(n)=\frac{n^{k}}{c} ; \quad c=\sum_{n=1}^{N} n^{k}
$$

where $n$ is the number of the interferer $n=1 \ldots N ., k$ is a variable that controls the relative scaling between interferers, and $c$ is a scaling factor to keep the total power constant.

Inclusion of the variable $k$ allows the skewing factor $\alpha$ to be used to allow a progression from all interferers being equal $(k=0)$, to one interferer being significantly dominant $(k>$ 20 ), while the total interfering power remains constant.

The normalized interferer power distribution for a set of five interferers, for various values of $k$ is shown in Fig. 7. The graph demonstrates that as $k$ is increased from 0 to 20 the power distribution among the five interferers progressively shifts from being all equal at $k=0$ to one interferer being dominant at $k=20$. The variable $k$ therefore represents various interferer power distributions among the interferers.

We will concentrate on the binomial symbol conditioned forms of the MCB and to investigate the performance of an optical system, for various power distributions among the interferers. A closer look at Fig. 7 demonstrates that the results expected between $k=0$ and $k=20$ should lie between the results obtained for the equally powered interferer case for five interferers equivalent to $k=0$ and a single interferer equivalent to $k=20$. 


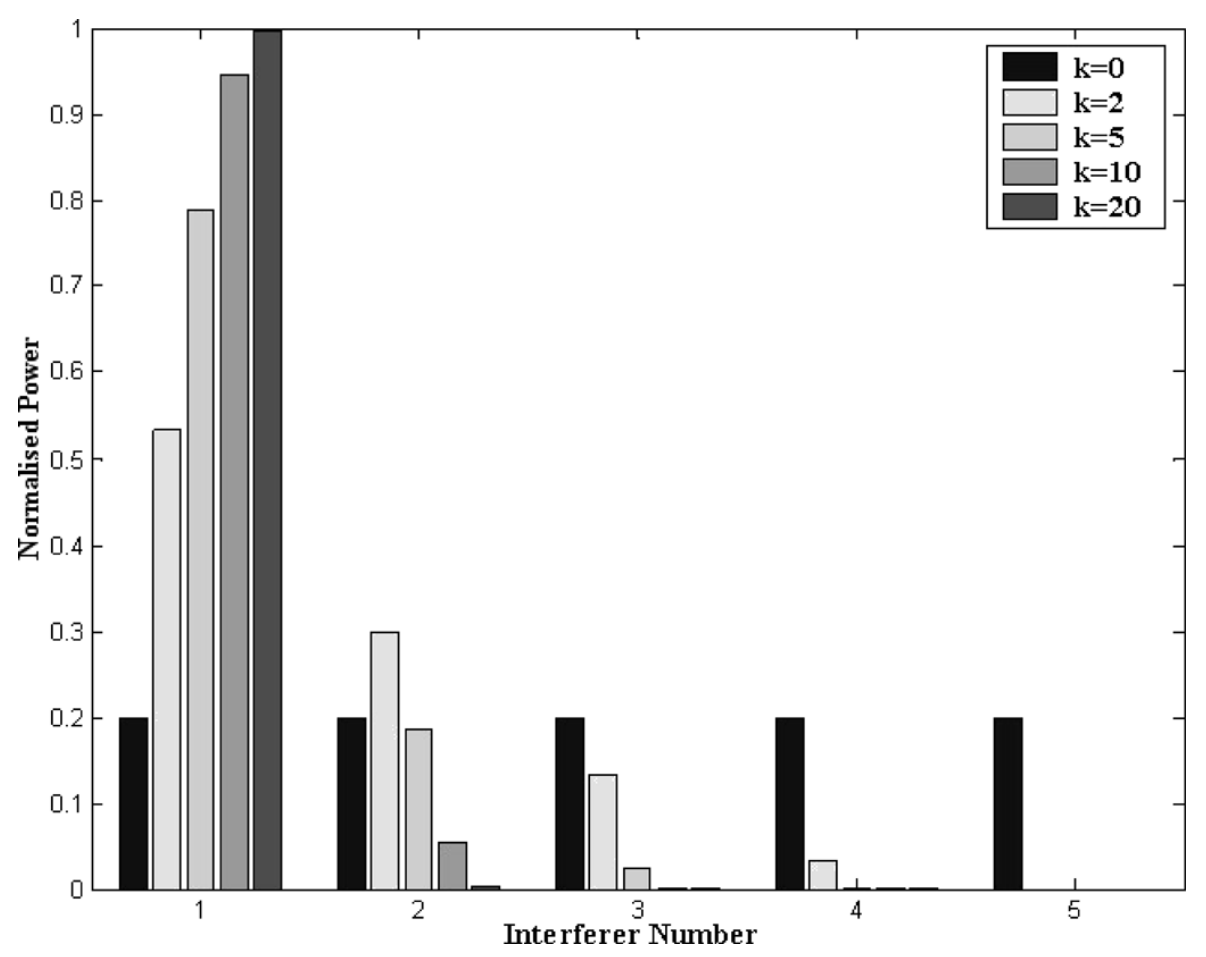

Fig. 7. Normalized interferer power $(N=5)$.

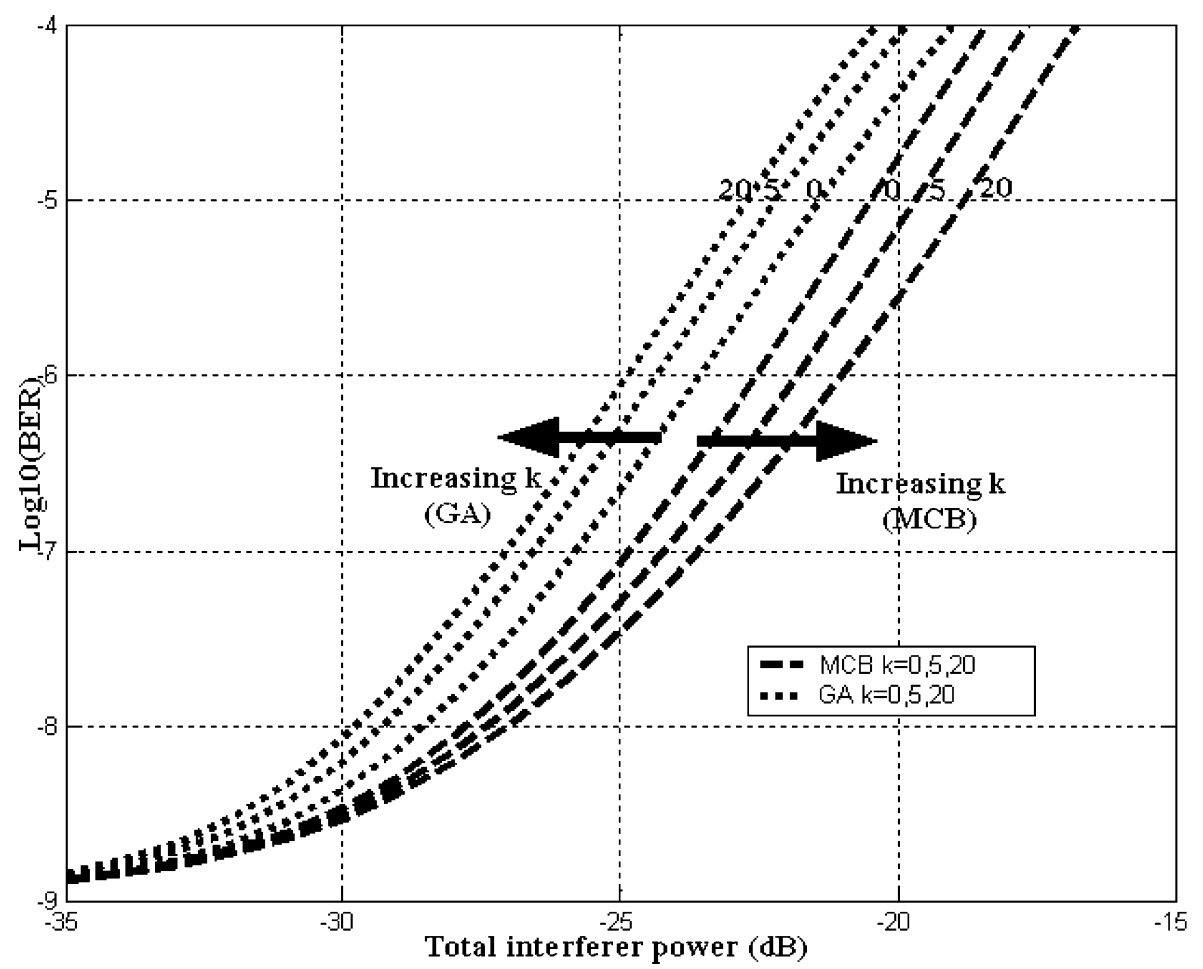

Fig. 8. BER for weighted interferers with $k=0,5$, and 20 calculated using the $\mathrm{GA}$ and $\operatorname{MCB}(N=)$.

In Fig. 8, the results predicted by the GA and the MCB are shown for five interfering terms. At $k=0$, the results estimated by both evaluation methods are reasonably close as this correspond to all 5 interferers being of equal power. The results then diverge as $k$ is increased, illustrating opposing trends where the GA predicting a worsening performance while the MCB predicts an improvement.
For $k=20$, results as given in Fig. 3. It is to be noted that the trends predicted as $k$ increases are contrary to those predicted in Section $\mathrm{V}$ for increasing $N$, due to the fact that the trend followed from $k=0$ to $k=20$ is equivalent to the trend followed in Fig. 2 from $N=5$ to $N=1$. The power penalty estimated using the various techniques (assuming five interferers) is illustrated in Fig. 9, which again shows the op- 


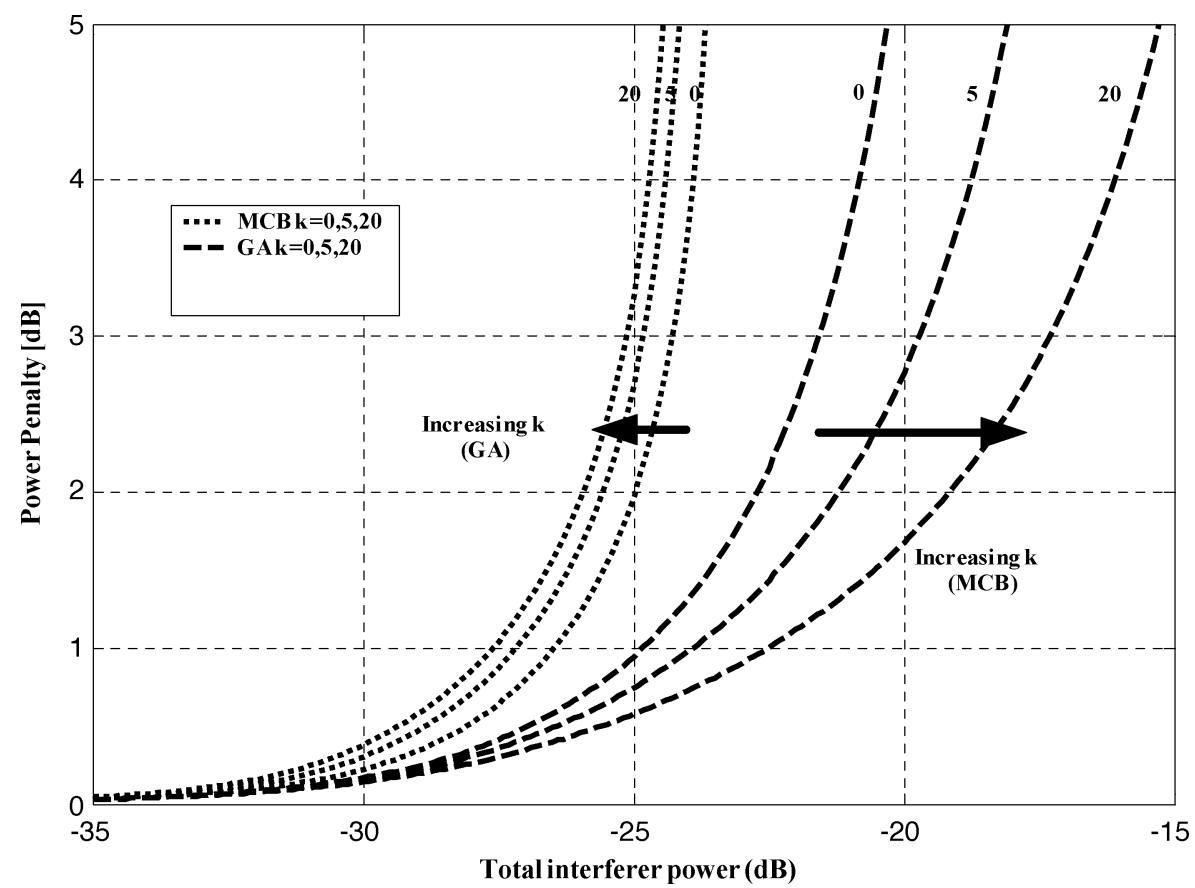

Fig. 9. Power penalty for unequally powered interferers.

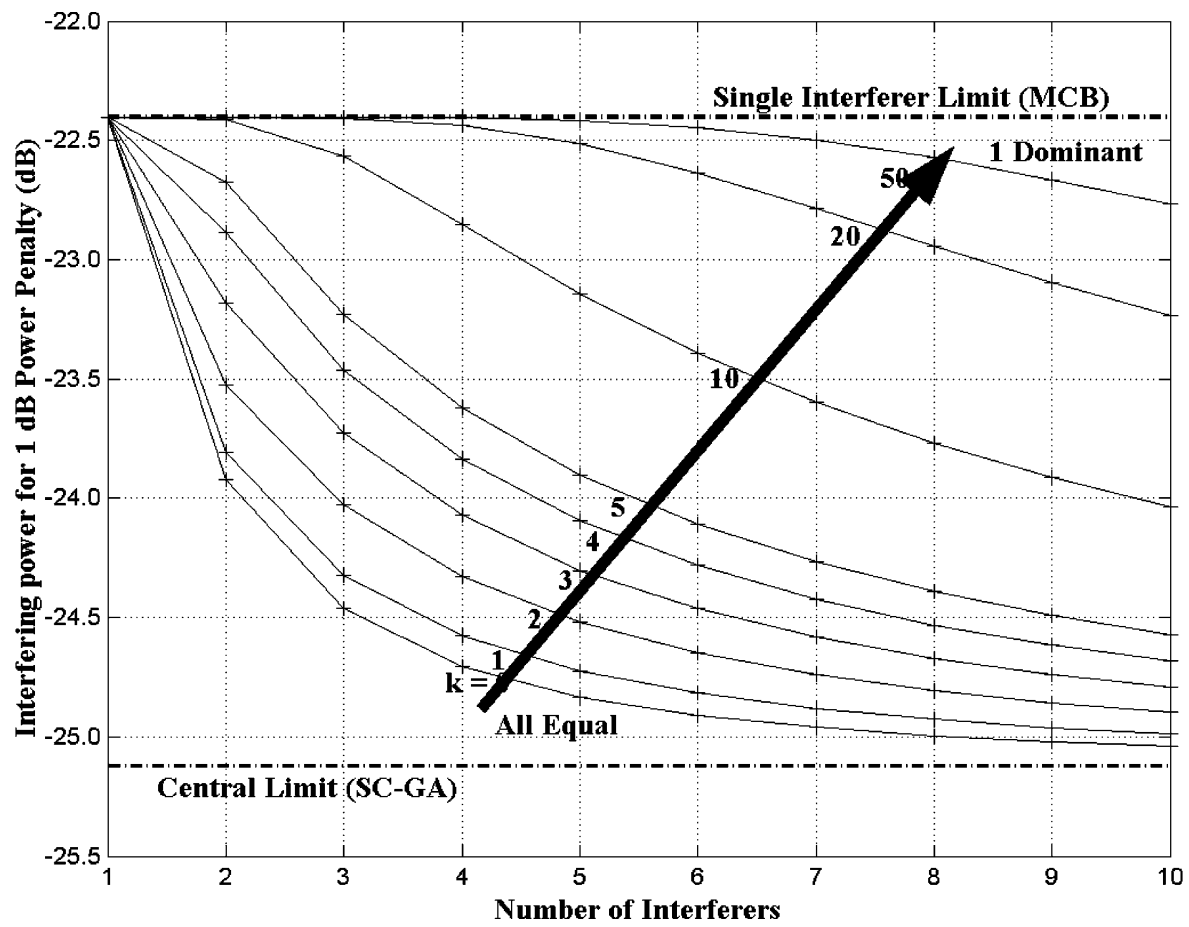

Fig. 10. Crosstalk power for 1-dB power penalty, showing the effects of scaling of the relative power of the interferers. The limit for the symbol-conditioned (SC-GA) and the single interferer limit from the MCB are presented.

posing trends predicted, with the more accurate results and true trend predicted by the MCB. It clearly illustrates the large variation in the penalty between that estimated using the GA and those estimated using the MCB. When investigating the impact of unequally powered interferers it is therefore necessary to utilize the MCB, because although the GA offers an easily obtained safe worst-case limit, it significantly overestimates the penalty incurred leading to inflated design margins. For example, this demonstrates that by simply calculating the total power and total number of contributing interferers, without consideration of the distribution of powers, the GA would suggest that a noise floor occurs at a maximum crosstalk isolation of around $-24 \mathrm{~dB}$. However, if the noise statistics were dominated by a single interferer contribution then the true noise floor would be in the region of $-15 \mathrm{~dB}$, an overspecification of $9 \mathrm{~dB}$, or nearly an order of magnitude.

With this in mind, the results of a selection of $k$ factors, for up to ten interferers, with a midway decision threshold are shown in a more condensed and directly applicable format in Fig. 10. It demonstrates the range of required crosstalk isolation for a 
1-dB power penalty at various levels of skew. Fig. 10 provides a better understanding of the interplay between the number of interferers, their relative normalized power distribution, and the normalized crosstalk interfering power required to cause a $1-\mathrm{dB}$ power penalty.

The results illustrate that we can define two limits for the acceptable crosstalk isolation to give a 1-dB power penalty. The lower limit is that defined by the GA for a large $N$, offering a worst-case prediction of the total allowable crosstalk for a 1-dB power penalty of approximately $-25.1 \mathrm{~dB}$. The upper limit is that defined by the MCB analysis of a single interferer setting the limit at $-22.4 \mathrm{~dB}$. The penalty range between the two limits is therefore $2.7 \mathrm{~dB}$. It is observed that for $N>10$ equally powered interferers, the result predicted is very close to that predicted by the symbol-conditioned GA (or central limit theorem).

\section{CONCLUSION}

In this paper, it has been demonstrated that the occurrence of interfering terms with unequal power distribution in optical networks can potentially lead to an overtly conservative estimation of the required crosstalk isolation. A thorough study of the impact of such interfering terms on the performance of an optical network was presented.

This study has again highlighted the inadequacy of the Gaussian approximation (GA) for accurate estimation of systems with a small number of interfering terms. An extension of this caveat to using the GA is proposed - that not only must there by a significant number of terms for this method to yield a reliable result, but that also the distribution of interfering power must not be dominated by a small number of interferers. Accuracy concerns in the use of this approximation are also highlighted by its prediction of inaccurate and counterintuitive trends when the distribution of interfering power is spread over differing numbers of interferers. The limited statistical knowledge involved (mean and variance only) causes trends to be produced that are, in fact, trends in the accuracy of the approximation rather than any "real" system trend.

It has been shown that for a 1-dB power penalty, the possible acceptable crosstalk covers a range of values -22.4 to -25.1 $\mathrm{dB}$ depending on the relative distribution of interfering power. The former value being the limit for a single or single dominant interferer and the latter being the central limit, on which a large number of similarly power interferers converge.

\section{REFERENCES}

[1] K. Inoue, "Suppression of influence of homowavelenght crosstalk in an optical add/drop multiplexing system by modulating LD light frequency," IEEE Photon. Technol. Lett., vol. 11, no. 9, pp. 1177-1179, Sep. 1999.

[2] W. D. Cornwell, I. Andonovic, A. Zadok, and M. Tur, "The role of thermal chirp in reducing interferometric noise in fiber-optic systems driven by directly modulated DFB lasers," J. Lightw. Technol., vol. 18, no. 2, pp. 154-160, Feb. 2000.

[3] K. Ho, "Analysis of homodyne crosstalk in optical networks using Gram-Charlier series," J. Lightw. Technol., vol. 17, no. 2, pp. 149-153, Feb. 1999.

[4] C. J. Rasmussen and P. Jeppesen, "New formulas for interferometric crosstalk penalty as a function of total crosstalk power, number of crosstalk contributions and signal extinction ratio," in LEOS Annu. Meeting 2000, vol. 1, 2000, pp. 25-26.
[5] M. Tur and E. L. Goldstein, "Dependence of error rate on signal-to-noise ratio in fiber optic communication systems with phase inducted intensity noise," J. Lightw. Technol., vol. 7, no. 12, pp. 2055-2058, Dec. 1989.

[6] J. E. Mitchell, P. M. Lane, and J. J. O'Reilly, "Statistical characterization of interferometric beat noise in optical networks," presented at the Conf. Optical Fiber Communication (OFC 1998), San Jose, CA, Feb. 1998, Paper WD3.

[7] H. Takahashi, O. Kazuhiro, and H. Toba, "Impact of crosstalk in an arrayed-waveguide multiplexer on $\mathrm{N} \times \mathrm{N}$ optical interconnection," $J$. Lightw. Technol., vol. 14, no. 6, pp. 1097-1105, Jun. 1996.

[8] J. L. Gimlett and N. K. Cheung, "Effects of phase to intensity noise conversion by multiple reflections on gigabit per second DFB laser transmission systems," J. Lightw. Technol., vol. 7, no. 6, pp. 888-895, Jun. 1989.

[9] S. D. Dods and R. S. Tucker, "Analysis of incoherent homodyne crosstalk in optical networks," J. Opt. Commun., vol. 23, pp. 178-181, 2002.

[10] D. A. Atlas, "Nonlinear optical crosstalk in WDM CATV systems," in Nanostructures and Quantum Dots/WDM Components/VCSEL's Microcavaties/RF Photonics for CATV HFC Systems, 1999 Dig. LEOS Summer Topical Meetings, 1999, pp. IV23-IV24.

[11] M. Tur and E. L. Goldstein, "Probability distribution of phase-induced intensity noise generation by distributed feedback lasers," Opt. Lett., vol. 15 , pp. 1-3, 1990

[12] J. E. Mitchell, P. M. Lane, and J. J. O'Reilly, "Comparative assessment of rigorous methods to evaluate interferometric noise in optical networks," in Proc. Int. Symp. Information Theory Its Applications (ISITA 2000), vol. 1, Nov. 5-8, 2000, pp. 405-408.

[13] J. J. O'Reilly and J. R. F. Da Rocha, "Improved error probability evaluation method for direct detection optical communications systems," IEEE Trans. Inf. Theory, vol. IT-33, no. 6, pp. 839-848, Nov. 1987.

[14] E. L. Goldstein, L. Eskildsen, C. Lin, and Y. Silberberg, "Polarization statistics of crosstalk induced noise in transparent lightwave networks," IEEE Photon. Technol. Lett., vol. 7, no. 11, pp. 1345-1347, Nov. 1995.

[15] C. Desem, "Optical interference in lightwave sub-carrier multiplexing systems employing multiple optical carriers," IEE Electron. Lett., vol. 24, no. 1, pp. 50-51, Jan. 7, 1988.

[16] - "Optical interference in subcarrier multiplexed systems with multiple optical carriers," IEEE J. Sel. Areas Commun., vol. 8, no. 7, pp. 1290-1295, Sep. 1990.

[17] P. J. Legg, M. Tur, and A. Andonovic, "Solution paths to limit interferometric noise induced performance degradation in ASK/direct detection lightwave networks," J. Lightw. Technol., vol. 14, no. 9, pp. 1943-1953, Sep. 1996.

[18] L. F. B. Ribeiro, J. R. F. Da Rocha, and J. L. Pinto, "Performance evaluation of EDFA preamplified receivers taking into account intersymbol interference," J. Lightw. Technol., vol. 13, no. 2, pp. 225-231, Feb. 1995.

[19] J. E. Mitchell, P. M. Lane, and J. J. O'Reilly, "Evaluation of extinction ratio induced performance penalty due to interferometric noise," IEE Electron. Lett., vol. 35, no. 12, pp. 964-965, Jul. 10, 1999.

[20] P. A. Rosher and A. R. Hunwicks, "The analysis of crosstalk in multichannel wavelength division multiplexed optical transmission systems and its impact on multiplexer design," IEEE J. Sel. Areas Commun., vol. 8, no. 6, pp. 1108-1114, Aug. 1990.

[21] J. J. O'Reilly and C. J. Appleton, "System performance implications of homodyne beat noise effects in optical fiber networks," Proc. Inst. Elect. Eng. . Optoelectronics, vol. 142, no. 3, pp. 143-148, Jun. 1995.

[22] C. F. C. Silva, R. Passy, J. P. Von der Weid, M. R. Jimenez, and A. O. Dal Forno, "Experimental and therorectical investigations of interferometric noise power penalties in digital optical systems," in IEEE MTT-S Int. Microwave and Optoelectronics Conf. (IMOC) 1999, pp. 562-564.

[23] L. Eskilden and P. B. Hansen, "Interferometric noise in lightwave systems with optical preamplifiers," IEEE Photon. Technol. Lett., vol. 9, no. 11, pp. 1538-1540, Nov. 1997.

[24] L. G. C. Cancela and J. J. O. Pires, "Rigourous evaluation of crosstalk requirements for large optical space switches based on directional couplers," presented at the Conftele 2001, Figueira da Foz, Portugal, Apr. 2001.

[25] J. J. O'Reilly and J. E. Mitchell, "Simplified Derivation of the Modified Chernoff Upper Bound,", to be published.

[26] V. K. Prabhu, "Modified Chernoff bounds for PAM systems with noise and interference," IEEE Tran. Inform. Theory, vol. IT-28, no. 1, pp. 95-100, Jan. 1982.

[27] C. Helstrom, "Approximate evaluation of detection probabilities in radar and optical communications," IEEE Trans. Aerosp. Electron. Syst., vol. AES-14, pp. 630-640, 1978.

[28] R. C. Boston, "Evaluation of Gram-Charlier coefficients," IEE Electron. Lett., vol. 7, p. 492, 1971. 
[29] K. Ho, "Analysis of co-channel crosstalk interference in optical networks," IEE Electron. Lett., vol. 34, no. 4, pp. 383-385, Feb. 1998.

[30] M. C. Jeruchim, P. Balaban, and K. S. Shanmugan, Simulation of Communications Systems (Application to Communication Theory). New York: Plenum, Dec. 1992.

[31] D. Remondo, R. Srinivasan, V. F. Nicola, W. C. van Etten, and H. E. P. Tattje, "Adaptive importance sampling for performance evaluation and parameter optimization of communications systems," IEE Trans. Commun., vol. 48, pp. 557-565, Apr. 2000.

[32] S. L. Danielsen, C. Joergensen, B. Mikkelsen, and K. E. Stubkjaer, "Analysis of interferometric crosstalk in optical switch blocks using moment generating functions," IEEE Photon. Technol. Lett., vol. 10, no. 11 , pp. 1635-1637, Nov. 1998.

[33] J. E. Mitchell, P. M. Lane, and J. J. O'Reilly, "Evaluation of interferometric noise from nonuniform interferers," presented at the 6th Eur. Conf. Network Optical Communications (NOC 2001), Ipswich, U.K., Jun. 25-28, 2001.

[34] P. J. Legg, "The impact of interferometric noise on the performance of optical communication networks," Ph.D. dissertation, Elect. and Elect. Eng. Dept., Univ. of Strathclyde, Strathclyde, U.K., 1995.

[35] J. E. Mitchell, "Beat noise and related phenomenon in optical networks," Ph.D. dissertation, Elect. and Elect. Eng. Dept., Univ. of London, London, U.K., 2000.

[36] C. J. Rasmussen, F. Liu, R. J. S. Pedersen, and B. F. Joergensen, "Theoretical and experimental studies of the influence of the number of crosstalk signals on the penalty caused by incoherent optical crosstalk," in Tech. Dig. IEEE/OSA Optical Fiber Communications Conf. 1999, vol. 1, Feb. 21-26, 1999, pp. 258-260.
[37] T. Kamalakis and T. Sphicopoulos, "Asymptotic behavior of in-band crosstalk noise in WDM networks," IEEE Photon. Technol. Lett., vol. 15, no. 3, pp. 476-478, Mar. 2003.

[38] L. Moura, N. Karafolas, A. Hill, P. Lane, and J. J. O'Reilly, "Statistical modeling of interferometric crosstalk in optical networks: the RACE II MUNDI network," in Proc. Conf. Networks and Optical Communications (NOC 1996), pp. 72-79.

J. C. Attard, photograph and biography not available at the time of publication.

J. E. Mitchell (S'97-M'00), photograph and biography not available at the time of publication.

C. J. Rasmussen, photograph and biography not available at the time of publication. 